\title{
Pseudomembranous colitis within radiotherapy field following concurrent chemoradiation therapy: a case report
}

This article was published in the following Dove Press journal:

OncoTargets and Therapy

18 January 2013

Number of times this article has been viewed

\author{
Bing-Jie Shen' \\ Shih-Chiang Lin ${ }^{2}$ \\ Pei-Wei Shueng 1,3 \\ Yueh-Hung $\mathrm{Chou}^{4}$ \\ Li-Ming Tseng ${ }^{5}$ \\ Chen-Hsi Hsieh ${ }^{1,6,7}$ \\ 'Division of Radiation Oncology, \\ Department of Radiology, Far \\ Eastern Memorial Hospital, Taipei, \\ Taiwan; ${ }^{2}$ Division of Oncology and \\ Hematology, Department of Internal \\ Medicine, Far Eastern Memorial \\ Hospital, Taipei, Taiwan; ${ }^{3}$ Department \\ of Radiation Oncology, National \\ Defense Medical Center, Taipei, Taiwan; \\ ${ }^{4}$ Department of Anatomical Pathology, \\ Far Eastern Memorial Hospital, Taipei, \\ Taiwan; ${ }^{5}$ Division of Colorectal \\ Surgery, Department of Surgery, \\ Far Eastern Memorial Hospital, Taipei, \\ Taiwan; 'Department of Medicine, \\ School of Medicine, National Yang- \\ Ming University, Taipei, Taiwan; \\ ${ }^{7}$ Institute of Traditional Medicine, \\ School of Medicine, National Yang- \\ Ming University, Taipei, Taiwan
}

Correspondence: Chen-Hsi Hsieh Division of Radiation Oncology, Department of Radiology, Far Eastern Memorial Hospital,

No 2I, Sec 2, Nanya S Rd, Banciao Dist,

New Taipei City 220, Taiwan

Tel +88628966 7000 ext 1033

Fax +886289660906

Email chenciab@gmail.com

Li-Ming Tseng

Division of Colorectal Surgery,

Department of Surgery, Far Eastern

Memorial Hospital, Taipei, Taiwan

No 2I, Sec 2, Nanya S Rd, Banciao Dist,

New Taipei City 220, Taiwan

Tel +886289667000

Fax +886289660906

Email liming.tt@msa.hinet.net
Abstract: Development of nonantibiotic-associated pseudomembranous colitis has been reported in patients receiving chemotherapy. Herein, we report a case of a 70-year-old man with diabetes mellitus and hypertension who received concurrent chemoradiation therapy after surgery for stage III pT3N1M0 rectal cancer. After completion of the therapy, the patient presented with a 2-week history of intermittent watery diarrhea (seven to nine times per day). However, the patient was afebrile and laboratory examination revealed no evidence of leukocytosis. Computed tomography disclosed inflammation of the sigmoid colon, infiltrative changes around the anastomotic site, and edematous changes straddling the serosal surface. Colonoscopic examination revealed multiple whitish patches within the radiation field, a finding suggestive of pseudomembranous colitis. No concomitant antibiotics were used during the period of concurrent chemoradiation therapy. Empirical oral metronidazole (500 mg every 8 hours) was administrated for 2 weeks. At the end of this treatment, stool culture was negative for Clostridium difficile. Physicians should be aware of the potential for the development of pseudomembranous colitis following concurrent chemoradiation therapy.

Keywords: diabetes mellitus, image-guided intensity-modulated radiotherapy, rectal cancer

\section{Introduction}

Clostridium difficile, a Gram-positive anaerobic bacillus, ${ }^{1}$ and its toxin can cause antibiotic-associated pseudomembranous colitis, a superficial necrosis of the colonic mucosa with an accompanying "pseudomembrane" (exudative plaque). ${ }^{2}$ However, pseudomembranous colitis due to $C$. difficile has also been diagnosed in patients who have not been exposed to antibiotic therapy, particularly in patients of older age, with diabetes mellitus (DM), renal failure, or intestinal obstruction, and those who have had intestinal surgery. ${ }^{3,4}$ Pseudomembranous colitis has also been reported in cancer patients after receiving antineoplastic chemotherapy without antibiotics. ${ }^{5}$

Herein, we report a case of nonantibiotic-associated pseudomembranous colitis that developed within the radiation field after postoperative concurrent chemoradiation therapy (CCRT) for rectal cancer in a patient with underlying DM. The investigation was reviewed and approved by the Institutional Review Board of the Far Eastern Memorial Hospital, Taipei, Taiwan.

\section{Case report}

A 70-year-old man with a history of DM and hypertension presented with stage III pT3N1M0 rectal cancer. A lower-anterior gross adeno-carcinoma resection was performed and adjuvant concurrent chemoradiation therapy undertaken from March 2010 
to mid-April 2010. Image-guided intensity-modulated radiotherapy was delivered to the surgical bed and pelvic nodal areas simultaneously at 54 Gy and 50 Gy, respectively, in 27 fractions over 6 weeks. The chemotherapy regimens included weekly bolus 5 -fluorouracil $\left(450 \mathrm{mg} / \mathrm{m}^{2}\right)$ and leucovorin $\left(30 \mathrm{mg} / \mathrm{m}^{2}\right)$. No concomitant antibiotics were used during the CCRT period.

After CCRT, the patient presented with a 2-week history of intermittent watery diarrhea (seven to nine times per day); however, he was afebrile and laboratory examination revealed no evidence of leukocytosis. Stool sample analysis revealed the presence of occult blood but no evidence of pus cells. Computed tomography (CT) disclosed inflammation of the sigmoid colon, infiltrative changes around the anastomotic site, and edematous changes straddling the serosal surface within the radiation field (Figure 1A and B). Colonoscopic examination revealed multiple white and yellow pseudomembranes on the wall of the rectum, as well as swelling, suggesting pseudomembranous colitis (Figure 1D). Histopathologic analysis of the biopsy specimens revealed partial or full thickness necrosis of the mucosa, glandular hypersecretion, and pseudomembranes composed of fibrin, mucus, and inflammatory cells with Gram-positive anaerobic bacillus, findings suggestive of pseudomembranous colitis (Figure $1 \mathrm{~F}$ and G). Anti-motility medication and empirical oral metronidazole (500 mg every 8 hours) were administered for 1 week as treatment for infectious diarrhea prior to stool testing. A stool sample was taken after antibiotic therapy and the results of the stool culture were negative for $C$. difficile. His symptoms subsided gradually and the number of yellow pseudomembranes decreased (Figure 1E). At 3-month follow-up, the patient was symptom free and abdominal CT revealed mild inflammatory changes around the anastomotic site (Figure 1C).

\section{Discussion}

C. difficile and its toxins are the most common causes of pseudomembranous colitis. ${ }^{6}$ Although disruption of the normal bowel flora due to antibiotic exposure is most often the cause of inflammation caused by $C$. difficile and its toxins, studies have shown that surgery following chemotherapy ${ }^{7}$ or chemotherapeutic agents ${ }^{5}$ can also cause extensive intestinal inflammatory changes by altering the normal bowel flora, leading to the growth of $C$. difficile and production of its toxins in the absence of antibiotic therapy.

However, to the best of our knowledge, the development of pseudomembranous colitis in the radiation field after
CCRT in patients who have not been exposed to antibiotics has never been reported. Hautmann et al noted that radiooncological patients were also patients at risk of $C$. difficile infection and that the incidence was $1.6 \%$, especially for head and neck cancer patients. ${ }^{8}$ Prendergast et al reported that irradiation-associated reductions in normal beef surface flora influenced survival of nonpathogenic Escherichia coli and pathogenic $E$. coli, suggesting that irradiation may affect the chemical or physical composition of flora. ${ }^{9}$ In addition, Rowe et al reported that free radical-induced oxidation of proteins and lipids may contribute to bacterial survival. ${ }^{10}$ This suggests that normal gastrointestinal flora may also be altered by irradiation. ${ }^{10}$

Development of pseudomembranous colitis has also been reported in patients of advanced age with DM after intestinal surgery in the absence of antibiotic treatment., One possible reason for changes in the normal flora in patients with diabetes is that diabetes results in decreased numbers of normal inhibitory bacteria in the oropharynx. ${ }^{11}$ Moreover, pseudomembranous colitis is also associated with high mortality and has a high rate of recurrence, especially in older and debilitated persons. ${ }^{12}$ In our patient, who had DM and had had intestinal surgery followed by CCRT, the CT (Figure 1B) and colonoscopic findings (Figure 1D) correlated with the radiotherapy target volume (Figure 1A), indicating that the pseudomembranous colitis within the radiation field after CCRT was caused by the synchronous effects of radiation therapy and chemotherapy.

The patient was prescribed oral metronidazole according to the CT scan (Figure 1B), pathologic (Figure 1F and G), and colonoscopic findings (Figure 1D) - initially without stool culture. $C$. difficile colitis may be diagnosed by sigmoidoscopy and/or stool tests. ${ }^{13}$ Bergstein et al ${ }^{14}$ reported that patients underwent lower gastrointestinal endoscopy with endoscopic confirmation of pseudomembranes but four (14\%) had only nonspecific colitis. Following pathologic and imaging findings for $C$. difficile colitis similar to our own, Kelly et al also reported a characteristic diagnosis. ${ }^{15}$ Oral metronidazole and vancomycin are equally effective in treating diarrhea caused by $C$. difficile infection. ${ }^{16}$ After receiving metronidazole, our patient's diarrhea improved and CT (Figure 1C) and colonoscopy (Figure 1E) images showed recovery. However, stool tests are still the most sensitive and specific for diagnosis of $C$. difficile infection. ${ }^{17,18}$ Therefore, we suggest that even when images and pathologic reports confirm pseudomembranous colitis, a stool test should be performed before treatment to confirm which antibiotics to administer. 


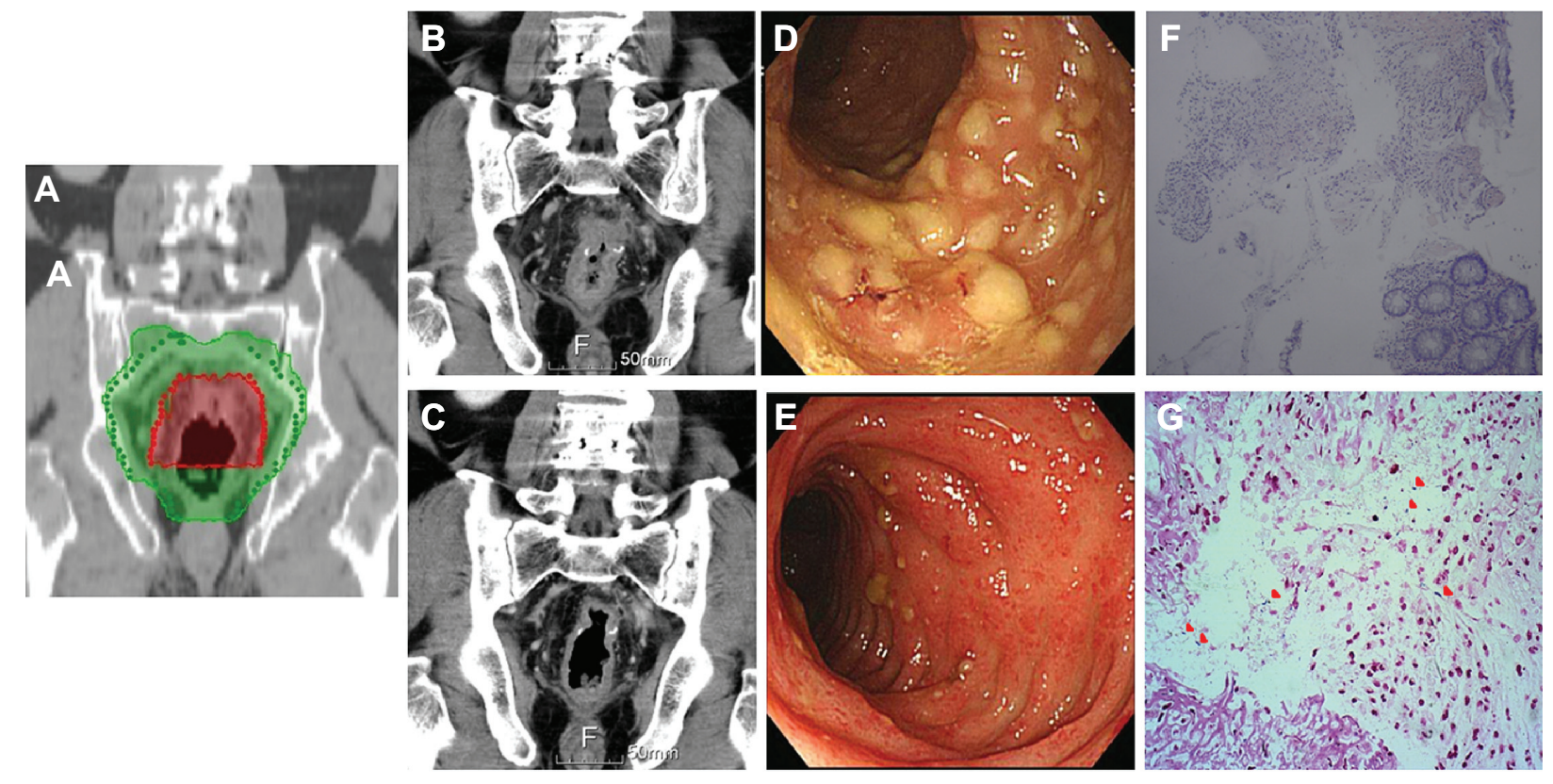

Figure I (A) The irradiation fields in radiation plan. Fifty-four Gray (Gy) and 50 Gy was delivered in red and green area, respectively. (B) The first evaluative CT scans after concurrent chemoradiation therapy. Inflammation of the sigmoid colon showing infiltrative changes around the site of anastomosis, edematous changes on the serosal surface, and marked thickening of the colonic wall. (C) The second evaluative CT performed at 3-month follow-up reveals mild inflammatory changes around the anastomotic site. (D) Endoscopic examination showed multiple white and yellow pseudomembranes on the wall of the rectum, as well as swelling, suggesting pseudomembranous colitis. (E) After a 2-week course of antibiotics, the number of pseudomembranes decreased and the severity of diarrhea gradually improved. (F) Histolopathologic analysis revealed partial or full thickness necrosis of the mucosa, glandular hypersecretion, and pseudomembranes composed of fibrin, mucus, and inflammatory cells (hematoxylin and eosin stain, $\times 100$ ), findings suggestive of pseudomembranous colitis. (G) Gram-positive bacilli were identified in the suppurative exudate over the colonic mucosa (red arrows, Gram stain, $\times 400$ ).

\section{Conclusion}

Elderly patients with DM who require CCRT after intestinal surgery are at risk of developing pseudomembranous colitis, especially if all three of these factors exist simultaneously. Stool tests should be routinely performed to establish whether there is pseudomembranous colitis, so that the appropriate antibiotics can be administered.

\section{Acknowledgment}

This study was supported by a grant from the Far Eastern Memorial Hospital (FEMH-2012-C-055).

\section{Disclosure}

The authors report no conflicts of interest in this work. Written informed consent was obtained from the patient to publish data and figures from his medical file.

\section{References}

1. Hall IC, O'Toole E. Intestinal flora in new-born infants with a description of a new pathogenic anaerobe, Bacillus difficilis. Arch Pediatr Adolesc Med. 1935;49(2):390-402.

2. Bartlett JG, Chang T, Taylor NS, Onderdonk AB. Colitis induced by Clostridium difficile. Rev Infect Dis. 1979;1(2):370-378.

3. Moskovitz M, Bartlett JG. Recurrent pseudomembranous colitis unassociated with prior antibiotic therapy. Arch Intern Med. 1981;141(5): 663-664.
4. Dosik GM, Luna M, Valdivieso M, et al. Necrotizing colitis in patients with cancer. Am J Med. 1979;67(4):646-656.

5. Anand A, Glatt AE. Clostridium difficile infection associated with antineoplastic chemotherapy: a review. Clin Infect Dis. 1993;17(1): 109-113.

6. Bartlett JG, Chang TW, Gurwith M, Gorbach SL, Onderdonk AB Antibiotic-associated pseudomembranous colitis due to toxin-producing clostridia. N Engl J Med. 1978;298(10):531-534.

7. Morales Chamorro R, Serrano Blanch R, Méndez Vidal MJ, et al Pseudomembranous colitis associated with chemotherapy with 5-fluorouracil. Clin Transl Oncol. 2005;7(6):258-261.

8. Hautmann MG, Hipp M, Kölbl O. Clostridium difficile-associated diarrhea in radiooncology: an underestimated problem for the feasibility of the radiooncological treatment? Radiat Oncol. 2011;6:89.

9. Prendergast DM, Crowley KM, McDowell DA, Sheridan JJ. Survival of Escherichia coli O157:H7 and non-pathogenic E. coli on irradiated and non-irradiated beef surfaces. Meat Sci. 2009;83(3): 468-473.

10. Rowe LJ, Maddock KR, Lonergan SM, Huff-Lonergan E. Influence of early postmortem protein oxidation on beef quality. J Anim Sci. 2004; 82(3):785-793.

11. Mackowiak PA, Martin RM, Smith JW. The role of bacterial interference in the increased prevalence of oropharyngeal gram-negative bacilli among alcoholics and diabetics. Am Rev Respir Dis. 1979;120(3): 589-593.

12. Moshkowitz M, Ben-Baruch E, Kline Z, Shimoni Z, Niven M, Konikoff F. Risk factors for severity and relapse of pseudomembranous colitis in an elderly population. Colorectal Dis. 2007;9(2):173-177.

13. Fekety R, Shah AB. Diagnosis and treatment of Clostridium difficile colitis. JAMA. 1993;269(1):71-75.

14. Bergstein JM, Kramer A, Wittman DH, Aprahamian C, Quebbeman EJ. Pseudomembranous colitis: how useful is endoscopy? Surg Endosc. 1990;4(4):217-219. 
15. Kelly CP, Pothoulakis C, LaMont JT. Clostridium difficile colitis. N Engl J Med. 1994;330(4):257-262.

16. Teasley DG, Gerding DN, Olson MM, et al. Prospective randomised trial of metronidazole versus vancomycin for Clostridium-difficileassociated diarrhoea and colitis. Lancet. 1983;2(8358):1043-1046.

17. De Girolami PC, Hanff PA, Eichelberger K, et al. Multicenter evaluation of a new enzyme immunoassay for detection of Clostridium difficile enterotoxin A. J Clin Microbiol. 1992;30(5):1085-1088.
18. Doern GV, Coughlin RT, Wu L. Laboratory diagnosis of Clostridium difficile-associated gastrointestinal disease: comparison of a monoclonal antibody enzyme immunoassay for toxins A and B with a monoclonal antibody enzyme immunoassay for toxin A only and two cytotoxicity assays. J Clin Microbiol. 1992;30(8):2042-2046.

\section{Publish your work in this journal}

OncoTargets and Therapy is an international, peer-reviewed, open access journal focusing on the pathological basis of all cancers, potential targets for therapy and treatment protocols employed to improve the management of cancer patients. The journal also focuses on the impact of management programs and new therapeutic agents and protocols on

\section{Dovepress}

patient perspectives such as quality of life, adherence and satisfaction. The manuscript management system is completely online and includes a very quick and fair peer-review system, which is all easy to use. Visit http://www.dovepress.com/testimonials.php to read real quotes from published authors.

Submit your manuscript here: http://www.dovepress.com/oncotargets-and-therapy-journal 